\title{
ANALYSIS ON MOBILE CLOUD SECURITY AND COMPARISON OF EXISTING MODELS
}

\author{
Soumya Ranjan Jena \\ Assistant Professor \\ Department of Computer Science \& Engineering \\ School of Computing \\ Vel Tech Rangarajan Dr. Sagunthala R\&D Institute of Science and Technology, Chennai, Tamil Nadu, India \\ soumyajena1989@gmail.com \\ Ashok Kumar Yadav \\ Assistant Professor \\ Department of Information Technology \\ Rajkiya Engineering College, Deogaon, Azamgarh, Uttar Pradesh, India \\ ashokyadav88.jnu@gmail.com \\ Shrikant Patel \\ Assistant Professor \\ Department of Computer Science \& Application \\ Delhi Skill and Entrepreneurship University, Delhi (Govt. of NCT of Delhi), India \\ patelshrikant@rediffmail.com \\ CH.M.H. Saibaba \\ Assistant Professor \\ Department of Computer Science \& Engineering \\ Koneru Lakshmaiah Education Foundation, Greenfields, Vaddeswaram, Guntur, Andhra Pradesh, India \\ saibaba.ch77@gmail.com
}

\begin{abstract}
:
The explosive emergence of Cloud Computing and its services are now everywhere in the World and it is one of the well-known technologies that we are using for both research and development activities nowadays. As small and medium organizations or companies are moving to Cloud Computing because as it supports rapid access to their applications and limits the cost of infrastructure. Cloud Computing for mobiles refers to Mobile Cloud Computing (MCC) as it is the collection of Mobile Computing and Cloud Computing to get one of the industry trend words and the major threads in the IT world since 2009. As business is growing using the computational resources on a pay as you go Model. This paper highlights the benefits and modern MCC uses and issues of MCC are briefly explained and also the security mechanism. MCC is investigating immense in IT due to anyplace whenever information gets to. Cell phones are empowered with rich client experience particularly, smartphones. Apple, Google, Facebook, and Amazon are the main four horsemen in the mobile world. That is the reason versatile distributed computing innovation is developing quickly among the clients and simultaneously, it presents new security dangers moreover. In MCC, a great deal of examinations is being done to annihilate the issues to make IT more solid and secure because all the more valuable information is put away in the Cloud condition. As the Internet-empowered cell phones, tablets keep on developing, online noxious dangers will keep on expanding in number to make more mind-boggling. Making sure information is more basic in the Mobile Cloud environment. In this paper, the working ideas of MCC and its different security issues and arrangements are given by analysts are broke down and also compare with different models.
\end{abstract}

Keywords: Mobile Cloud Computing, Mobile Cloud Security, Mobile Cloud Networking. 


\section{Introduction}

MCC applications are internet-based and multi-platform that may be installed on differing kinds of mobile devices with varied operational systems. So, for example, if a user enters a photograph on a Cloud app put in on her Smartphone, she will access that image on the same Cloud app put in on her pill and/or portable PC that has totally different operational systems.

Today, there is a good vary of Cloud apps: instant electronic messaging (WhatsApp, Viber), taking notes (Evernote), photo sharing (Snapchat), and file storage and sharing (Google Drive, Dropbox), etc. Even though MCC fulfills several mobile device users' desires and provides tremendous deserves, they arouse privacy considerations regarding revealing personal info to those apps.

On the opposite hand, Cloud Computing literature highlighted many blessings of Cloud apps: (a) period of time application response; (b) increasing the mobile device performance; (c) scalability; (d) reliability; (e) less energy consumption; (f) a lot of knowledge storage capacity; (g) enhancing process power. Thus, these prices and advantages of revealing personal info to MCC apps create a quandary for individuals to use these apps.

This study has many vital contributions to privacy and Cloud Computing literature: 1 st, the majority of privacy studies regarding MCC apps read the development from a technical perspective to debate how MCC suppliers will use new privacy practices. However, we tend to believe that as long as MCC suppliers do not recognize what factors influence their customers' privacy considerations regarding victimization MCC apps, a group of individuals still don't use such applications whether or not MCC suppliers acquire the newest privacy and security solutions. Since behavioral aspects of MCC apps privacy have received very little attention, this study opens new views on individuals' privacy considerations regarding victimization MCC apps.

Second, it's prompt that privacy be examined in several contexts so individuals' attitudes towards business apply are utterly understood. MCC apps incorporate plenty of disparate applications and have distinct options, but the previous studies mentioned either privacy on the web that's a really broad thought and will not think about specific options of different technologies, or privacy of specific applications (i.e., location-based service mobile applications) that's a really slender concept. The options of MCC apps are hybrid and mix those of net and mobile applications while adding options that are specific to privacy analysis. This study extends privacy literature by securitizing privacy perceptions regarding all kinds of MCC apps and provides insight into the privacy considerations that ought to be taken into consideration by MCC applications providers to develop their applications.

\section{Research Challenges}

The key exploration difficulties can be represented as an expansive examination of security issues in MCC as it has a few preferences for both versatile clients and Cloud specialist co-ops, it faces a few difficulties that make it more confused than customary Cloud Computing. We have written this research paper as given as follows. Section 3 highlights the literature survey. Section 4 and 5 highlights the architecture of MCC and Mobile Cloud security concerns. Section 6 limelight the various security concerns in MCC. Comparison of existing models discusses in Section 7. Mobile Cloud networking is given in Section 8. A tabular representation of existing model comparisons has been narrated in Section 9. Finally, Section 10 and 11 discuss about the analysis and the conclusion and future research scope respectively.

\section{Related Research Works}

The security of utilizations manages the assurance of mobile applications or mobile application models, which utilize the Cloud assets to offer better types of assistance for mobile clients in the MCC condition. Our early works [19-29] was completely based on Cloud based environments to find out different parameters. Kun Yang et al. have discussed [2] Mobile Cloud Computing and networking. Mobile Cloud Computing implications and challenges [3] are described by M. Rajendra Prasad and Ronnie D. Caytiles et al. [4] have described the security concerns for MCC. Whereas in [5] authors have explained the MCC security considerations.

Authors in [6] [7] have described the framework for secure data processing in MCC. Authors in [30] have devolved an advanced Q-MAC which is basically used for optimal resource allocating for dynamic application in Mobile Cloud Computing using QoS with cache memory. L. Pallavi et al. [31] [32] have developed two algorithms i.e. ERMO Algorithm and BTS algorithm for an energy efficient mobility management in Mobile Cloud Computing system for 5G heterogeneous network. They have also in [35] described about mobility management challenges and solutions in Mobile Cloud Computing system for next generation networks. In [33] authors have narrated PIOCM which extensively defines the properly identifying optimized Cloudlet in Mobile Cloud Computing. In [34] Sairam, V.P.N et al described a User 
privacy-preserving encryption strategy out sourcing data in Mobile Cloud Computing. Nalajala, S. et al. [36] have described a light weight secure data sharing scheme for Mobile Cloud Computing. Ravindranath, $\mathrm{K}$. at al in [37] narrated a Dynamic optimization local search offloading algorithm for Mobile Cloud Computing. Hoang T. Dinh et al in [38] have extensively studied the MCC architecture, applications and approaches.

\section{Architecture of MCC}

From the concept of MCC, the general architecture of MCC is shown in Fig. 1. In this architecture, mobile devices can get to Cloud benefits in two different ways, i.e., through portable base stations e.g., base handset station (BTS) and passageway, or satellite. This sets up and controls the associations and useful interfaces between the systems and cell phones. Portable clients' solicitations and data (e.g., ID and area) are sent to the focal processors that are associated with workers giving versatile system administrations. Here, versatile system administrators can offer types of assistance to portable clients (for verification, approval, and bookkeeping) in view of the home specialist (HA) and supporters' information put away in data sets.

From that point forward, the supporters' solicitations are conveyed to a Cloud through the Internet. In the Cloud, Cloud regulators measure the solicitations to give versatile clients the comparing Cloud administrations. These administrations are created with the ideas of utility registering, virtualization, and administration arranged design (e.g., web, application, and information base workers).

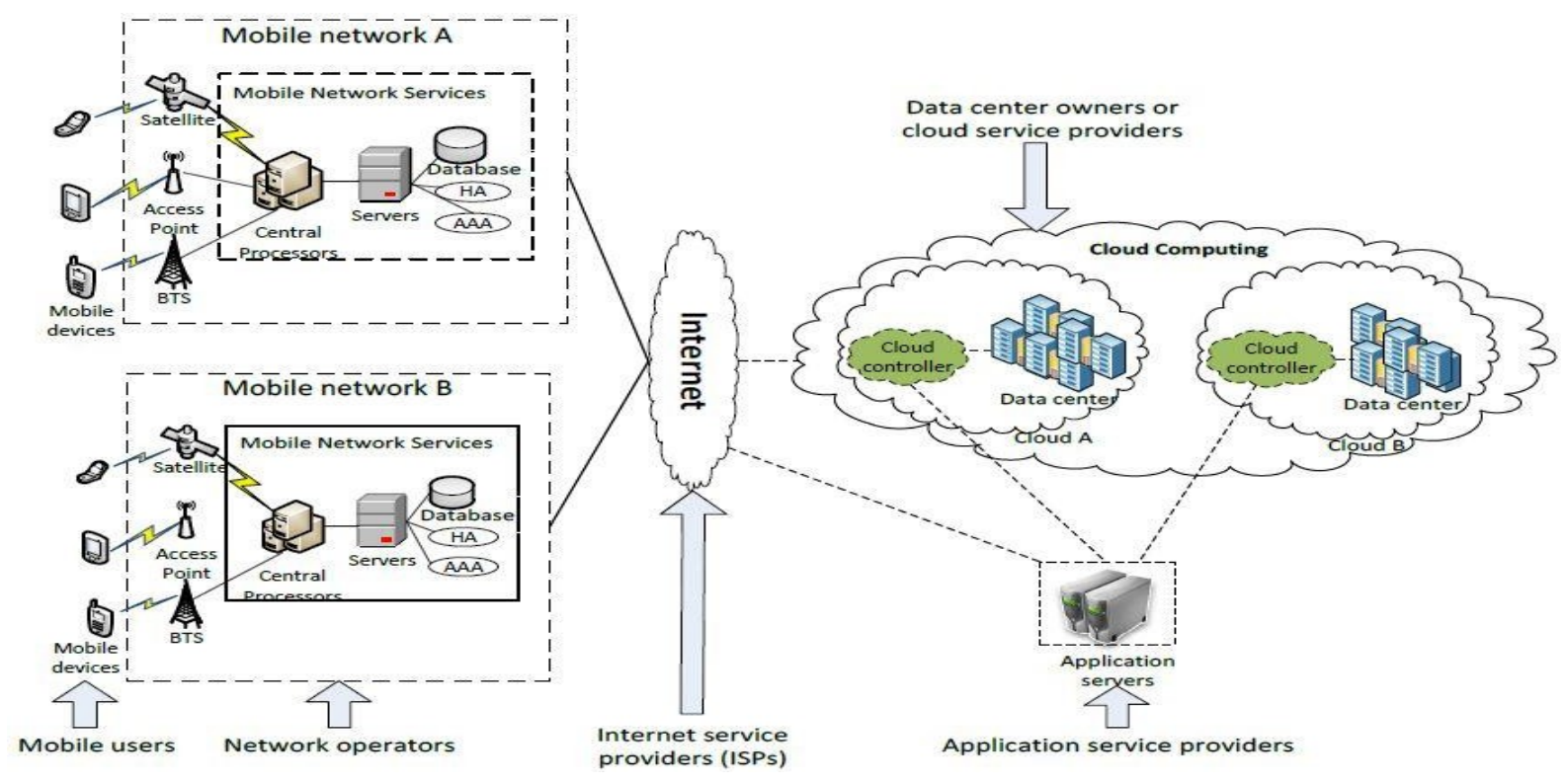

Fig.1 Mobile Cloud Computing Architecture [38]

\section{Mobile Cloud Security}

MCC conveys with it 2 significant parts: one is Cloud and another is portable devices. The last part and a couple of parts of the past have been referenced inside the previous areas. In our survey it has been referenced that to understand a protected mobile Cloud structure, in elective words, a safe mobile Cloud cannot be accomplished while not introductory creation certain a safe Cloud condition. For this reason, have we tend to referenced widely gauges required to ensure the security of the Cloud. This area examines the security issues/difficulties of the mobile Cloud that we will, in general, accept joined with the data assembled from the audits allotted on Cloud security can ensure achievement in arranging a safe portable Cloud engineering. Fundamentally the wellbeing issues in portable distributed computing are identified with (1) security issues in the Cloud, (2) security of the phone and (3) the security of the line between the Cloud assets.

\section{Security Concern}

In this space of Mobile Cloud Computing security problems arises in 2 totally different components one belongs to mobile phones and mobile network security and another is security on Cloud i.e. Cloud security. 


\subsection{Cloud-based Security Concern}

Cloud gives stockpiling and calculation and furthermore besides the data keeps on the Cloud needs security [13] which depicts the Cloud security front in 3 little print. First is to remain the data taken care of inside the Cloud secure which proposes the information kept by the customer couldn't be gotten to by the untouchable so for that the information is taken care of in mixed kind on the Cloud in any case that data is also known to the Cloud supplier in Associate in Nursing decoded kind as encryption is performed by the Cloud suppliers only with the objective that they should be solid.

\subsection{Securing Mobile Cloud Requirements}

1. Integrity: In integrity the information keeps on the Cloud by the client through the cell phone should be incorporated. Clients should capture any place the information is solid and who will get to it and each entry should be approved consequently therefore no run of the secret data is finished.

2. Authentication: In authentication the major issue when compact contraptions purchaser gets to the data keep on Cloud each passage ought to be ensured so the customer will get to data related with them solely no unapproved get to ought to be appropriate and this should be conceivable by various check instruments like giving login ids passwords, pins to particular customer to affirm their character which grants them to incite admittance to their information ardently.

3. Digital rights management: Computerized media are getting pilfered just because of their essence on Cloud obviously. Media like recordings, pictures, sound, and eBooks are wildly gotten to with the goal that they should be whole in encoded kind so no theft of the individual media fundamental to the client will get an irresistible specialist on the net unlawfully.

\section{Comparison of Existing Models}

\subsection{Smartlab Architecture}

SmartLab is an experimental test bed of about forty plus real android smartphones and emulated devices running android operating system. This system is deployed at the Computer Science department infrastructure building at the University of Cyprus. This enables the registered users to achieve the following tasks, once they have to download and install the apk or the executable file on their device.

- Upload and install apk file on a variety of android running devices,

- Capture output

- Rebooting of devices

- Create jobs which are interactive using Monkey Runner Scripts

- $\quad$ Full remote device interaction, and many more

The basic aim of SmartLab architecture is to facilitate and promote research in a network of mobile Cloud programming environments and to improve communication protocols and system designs and other applications. This comprises of an air conditioned laboratory with a connected network of forty plus smartphones featuring Wi-Fi coverage. These smartphones are mounted to a VMware Vcenter remotely located in a cluster of rooms using local proxies. Android ADB server is used to instruct and execute all the commands throughout the connected network of smartphones. And all these states of connections are maintained in a MySQL database.

\subsection{Smartlab Work Flow}

The workflow of SmartLab architecture is divided into 3 phases with each consisting of several steps in between the individual deployment i.e. resource allocation, document transfer and cooperate with devices. 


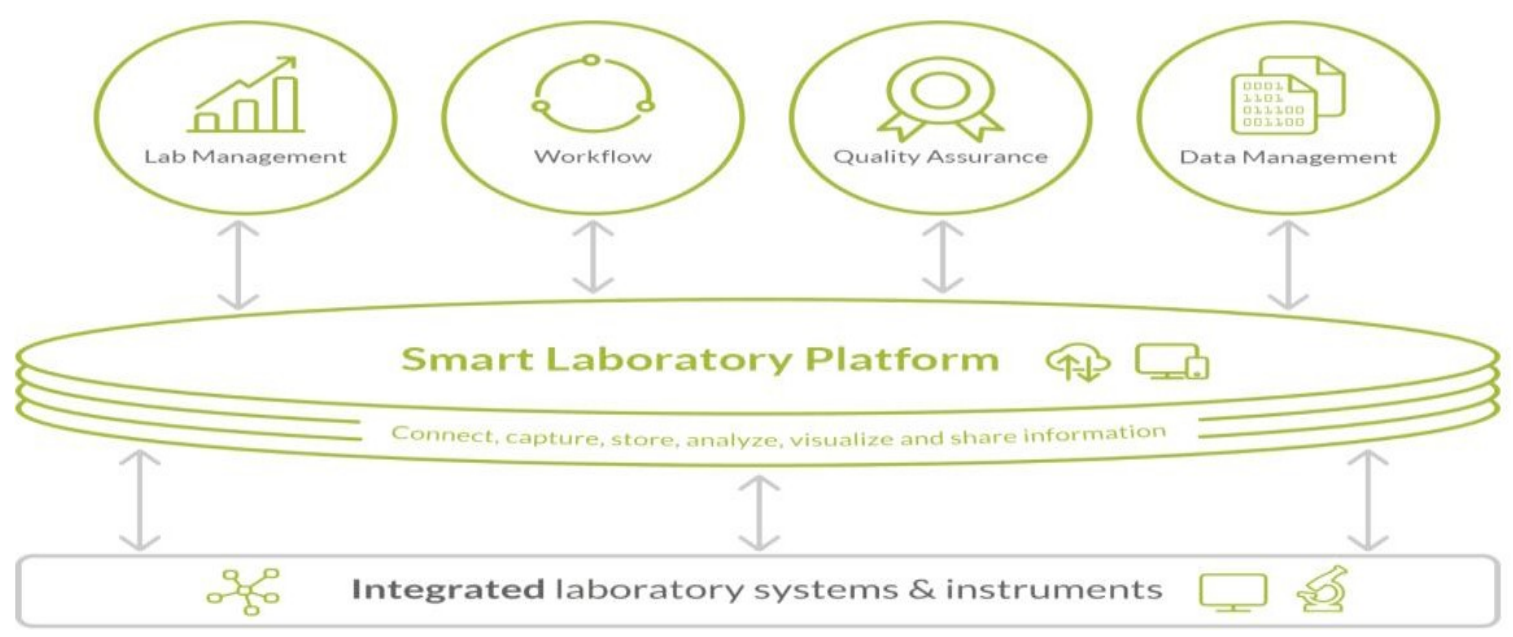

\section{Operations}

.Fig. 2 Smartlab Workflow

Coming up next are the utilization of the design:

- SmartTrace : This is a structure that is publicly supported which is utilized for executing conveyed likeness looking through questions dependent on the directions that are put away on the associated system of the cell phones

- SmartP2P: This is a novel system intended for looking through articles, for example, pictures, recordings, and different documents. These are caught by the current client in a network that is worried about client security.

\section{Future Work}

- $\quad$ SmartLab is open to the public for registration

- $\quad$ New Features such as Web 2.0 GUI, Eclipse Plugin, Crowd sourced Platform

- Scientific publication summarization of SmartLab Experiments

\section{Mobile Cloud Networking}

In recent times, MCC is becoming very dominant in the driving of the IT industry. The Mobile Cloud can be defined as the combination of mobile device, network, storage and non-localized computing, with several features like on-demand and pay as you go service.

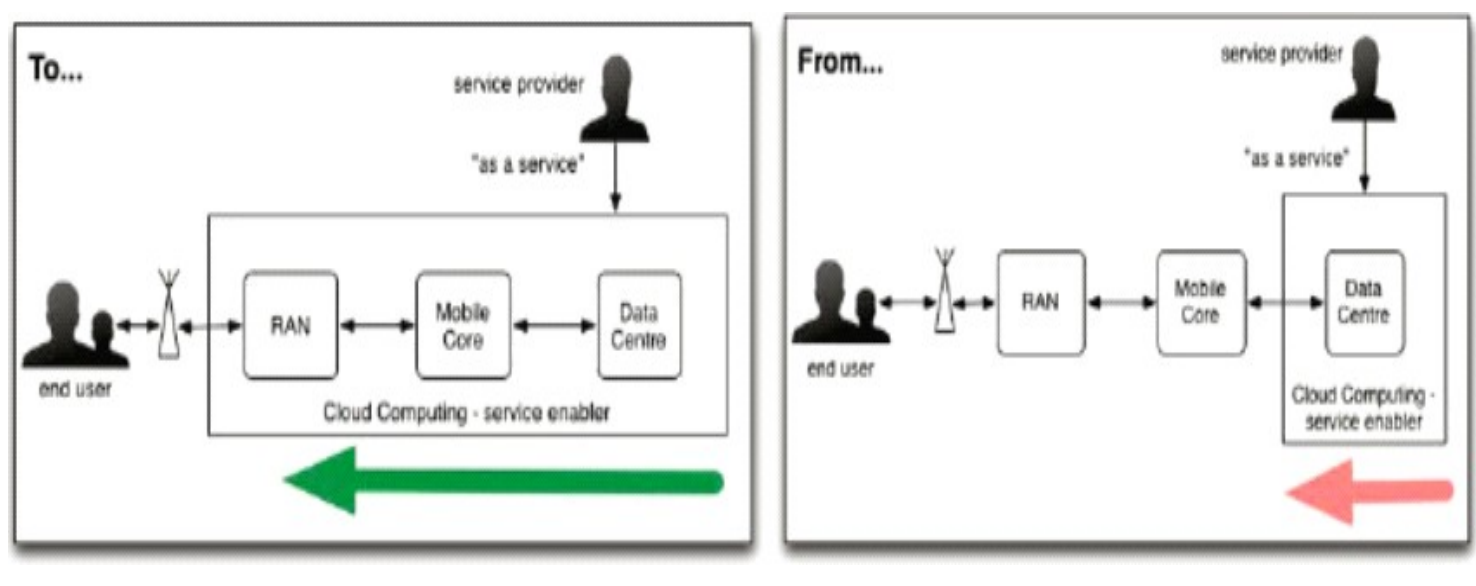

Fig.3 Mobile Cloud Networking

One of the significant issues of Cloud Computing is the development of programming businesses that isn't comprehended by telecom specialists. 


\subsection{Objective of Mobile Cloud Project}

- $\quad$ Prototype creation using novel mobile network architecture and technologies using the proof-of concepts.

- $\quad$ Expend the support and elasticity of novel mobile services that are currently existing over the globe.

The investigation, implementation, and assessment of the mechanical establishment for such a framework is the primary objective of Mobile Cloud Networking. This will bolster continuous help and on-time execution which is more productive. These can be founded on a baseband unit parade and QoS Control. This is going to run on an upgraded portable Cloud stage utilizing the product accessible equipment.

This can only be achieved by a greater unit of a decentralized procession of the data which is purely based on the load balancing factor. This also provides Wireless, mobile core, and End to end SLAs and through the follow me Cloud concept. This project is leading the technical Part of the project is controlled by SAP and ZHAW.

\subsection{Mobile and Distributed Computing (MDC Lab)}

As we are discussing on MDC, we first know what Mobile Computing and Distributed Computing is. MDC which targets on three things that are architectures, protocols and platforms in mobiles and distributing computing. The gathering plans to create calculations, apparatuses, and advancements which offer vitality productive, blame tolerant, adaptable, secure, and elite registering administrations. The exploration interests of the gathering incorporate portable processing, distributed computing, matrix figuring, and substantial scale disseminated frameworks, universal registering, mist figuring, superior frameworks for enormous information examination, green processing, versatile/specially appointed systems, and security. MDC is as of now taking a shot at numerous examination extends and teaming up with research gatherings/labs around the globe. Mobile Computing is becoming very dominant in the driving of the IT industry. In 1990, mobile phones are meant for voice calls and messages. Mobile Computing can be defined as the combination of mobile device, network, storage and non-localized Computing.

The basic aim of Mobile Computing is to promote research in the network of mobile Cloud programming environments.

\subsection{Distributed Computing}

It is a term used to solve big problems from many computers. One method to solve big problems by using a supercomputer. As supercomputers are very expensive so many researchers do not use it. Another way to solve the problem to split the big problem into smaller problems. An example where Distributed Computing is used is the Great Internet Mersenne Prime Search. Another way to define Distributed Computing that it is a field of software engineering that reviews circulated frameworks. A distributed system is a network that consists of self-ruling PCs that are associated with utilizing a conveyance "middleware ". It allows resource sharing and allows the users with a single and integrated coherent network.

The advantages of Distributed Computing are:

1. Telecommunication network

2. System applications that are the internet and distributed system.

Future Work for Distributed Computing are:

1. Move to micro services.

2. Other teams and companies run and manage our services and infrastructure.

This makes the future of Mobile and Distributed Computing looks increasingly exciting.

\subsection{Mobile Cloud Computing (MobCC Lab)}

In recent times the Mobile Cloud Computing is becoming very dominant in the driving of the IT industry. Mobile Cloud, as the name, suggests that it is the combination of Mobile device + Network + Storage + NonLocalized Computing. Have various features like On-demand and pay as you go service. Mobile Cloud 
Computing Lab is deployed at the Faculty of Computer Science and Information Technology at the University of Malaya.

The various advancements in MobCC lab are given as follows:

1. Advancement of novel technologies and structures utilizing the model with the verification of-idea to change the current, versatile structure and change to entirely Cloud-based portable frameworks.

2. The extension of Cloud Computing to support the elasticity and provisioning of novel mobile services.

3. Creation of the concept of business support systems that are fully embraced with mobile Cloud notions.

\section{InIT Cloud Computing Lab (ICCLab)}

ICCLAB is an examination lab expanding and driving the Cloud figuring exploration and instruction. This lab research is driven by Prof-Dr. Thomas Michael Bohnert and it is useful under the Service Engineering gathering of Zhaw's InIT (Institute of Applied Information Technology) fuse with the Splab. It comprises of various foundations, levels of understanding, and nationalities. An exploration group is a functioning gathering whose space exists in Swiss and EU research fields and furthermore critical two-sided exercises with neighborhood Swiss organizations.

\section{ICC LAB FEATURES}

- It has an all-around thought and creative examination approach.

- It is fundamentally concentrating on Education.

- It is dynamic in MCC ventures.

- It produces open-source free software.

Mobility, ubiquity, and anyplace, whenever, anything joined with on-request benefits is the forces of the Cloud. Mobile and Cloud Computing are the two transformations driving and pushing its industry in recent and upcoming years.

\section{Objectives}

- To build up a worldview and advancements, utilizing calculated models, to manage clients from current mobile structures to completely Cloud-based mobile frameworks.

- To stretch out Cloud Computing to help client's on-request administration and adaptability of offering types of assistance.

- To grow new ideas for operational and business emotionally supportive networks that completely rise the progression of mobile Cloud.

\subsection{Mobile and Cloud Lab}

Mobile and Cloud Computing Laboratory is arranged at the Institute of Computer Science, Faculty of Science and Technology, University of Tartu. It oversees exploration and instructing in the field of portable registering and distributed computing territory. It for the most part centers on the business inclining points like Cloud Computing, versatile application advancement, mobile Cloud, mobile web benefits, the Internet of Things, and are moving towards logical registering and undertaking and modern applications to the Cloud.

\section{Research works}

- Cloud Computing-The research objective here is to mull over the development of enormous business applications to the Cloud and to think about their execution on the Cloud.

- Scientific Computing On Cloud-The research objective here is to consider the development of intelligent figuring applications to the Cloud and to diminish the mass weight on these applications and counts to Cloud Computing structures like MapReduce.

- Mobile computing-This research manages to create mobile applications for different stages and gadgets (for example, Android, iOS, Windows Phone 7, and so forth). It additionally manages to secure and using sensor information in building and dealing with applications for various areas. Mobile Cloud-The objective of this research is to research how to proficiently and achieve extreme use of Cloud assets inside a Cloud application (otherwise known as mobile Cloud applications). 
- Mobile Web Services- This research topic manages the conjuring, provisioning, revelation and combination of web administrations from PDAs, in creating mobile applications.

- Internet of Things-The objective of this research is to beaten the difficulties of digital physical frameworks in the Internet of Things. The difficulties incorporate interoperability, independent machine-to-machine correspondence, programmed design, vitality effectiveness, dependability, and so on.

\section{Existing Models Comparison}

\begin{tabular}{|c|c|c|c|c|}
\hline Models & Security & Cost & Future Scope & Scalability \\
\hline $\begin{array}{c}\text { Smart lab } \\
\text { Architecture }\end{array}$ & $\begin{array}{l}\text { Smart trace and } \\
\text { Smartp2p Are Used } \\
\text { for Protecting the } \\
\text { Privacy Of User }\end{array}$ & Low & $\begin{array}{c}\text { Working on } \\
\text { Web2.0 GUI And } \\
\text { Eclipse Plugin }\end{array}$ & High \\
\hline $\begin{array}{r}\text { Mobile Cloud } \\
\text { Networking }\end{array}$ & $\begin{array}{l}\text { Implements of Various } \\
\text { Security Algorithms } \\
\text { for Seamless } \\
\text { Experience }\end{array}$ & Medium & $\begin{array}{c}\text { Working to Minimize } \\
\text { Data Loss }\end{array}$ & Low \\
\hline $\begin{array}{l}\text { Mobile And } \\
\text { Distributed } \\
\text { Computing }\end{array}$ & $\begin{array}{c}\text { Distributed System are } \\
\text { Used as They are More } \\
\text { Secure }\end{array}$ & High & $\begin{array}{c}\text { Working to Provide } \\
\text { Low Infrastructure } \\
\text { Cost }\end{array}$ & High \\
\hline $\begin{array}{c}\text { Mobile Cloud } \\
\text { Computing }\end{array}$ & High & High & $\begin{array}{l}\text { Working to Minimize } \\
\text { Data Fluctuation }\end{array}$ & Medium \\
\hline $\begin{array}{c}\text { Init Cloud } \\
\text { Lab }\end{array}$ & $\begin{array}{l}\text { Peer Too Peer } \\
\text { Encryption }\end{array}$ & Medium & $\begin{array}{l}\text { Stabilizing and } \\
\text { Initiating VM } \\
\text { Migration }\end{array}$ & Medium \\
\hline $\begin{array}{r}\text { Mobile And } \\
\text { Cloud Lab }\end{array}$ & $\begin{array}{c}\text { Various } \\
\text { Encryption } \\
\text { Algorithms Used } \\
\text { At Both Ends. }\end{array}$ & Low & $\begin{array}{c}\text { Image Sensor and } \\
\text { Tools To Direct } \\
\text { Migrate On Cloud }\end{array}$ & Medium \\
\hline
\end{tabular}

\section{Analysis}

Cloud Computing is that the model offers a couple of sorts of help and now used for security works conjointly. There exist genuine issues that choice for worry about this new variety of innovation anyway some of these contemplations are unimportant bits of gossip with none premise of defense. One of the significant concerns alluding to this imperative subject of intrigue (Mobile Cloud Computing) is that of security (KPMG, 2010), that this examination has focused on. In leading this investigation, we will in general have made an undertaking to explain the wellbeing challenges that plague this developing innovation and have gone a stage extra to proffer achievable moderation plans or answers for rib these security contemplations. Through an escalated audit of significant writing, we have been prepared to get statistical data points identifying with the expected dangers of interest in Mobile Cloud Computing. we will, in general, didn't complete our existence discovering drive on just information accumulated from instructional exercise examination or materials. Outfitted with information from trusty gatherings we tend to have the option to set up what some of the significant contemplations are tied in with receiving versatile Cloud Computing. With an unmistakable order knowledge, we will in general proceed with investigating and causing out polls to detect some of the courses through that the setup contemplations are regularly settled subsequently on blessing a feasible response to reestablishing trust in portable distributed computing, considering the tremendous edges of Mobile Cloud Computing and Cloud Computing; a premier examination group has tipped distributed 
computing that will be that the bedrock for Mobile Cloud Computing due to the first among the most elevated 10 most significant innovations with an improved possibility with time..

Truly, there exist genuine issues that choice for worry about this new variety of innovation anyway some of these contemplations are unimportant bits of gossip with none premise of defense. One of the significant concerns alluding to this imperative subject of intrigue (Mobile Cloud Computing) is that of security (KPMG, 2010), that this examination has focused on. In leading this investigation, we will in general have made an undertaking to explain the wellbeing challenges that plague this developing innovation and have gone a stage extra to proffer achievable moderation plans or answers for rib these security contemplations. Through an escalated audit of significant writing, we have been prepared to get statistical data points identifying with the expected dangers of interest in Mobile Cloud Computing. We will, in general, didn't complete our existence discovering drive on just information accumulated from instructional exercise examination or materials. Outfitted with information from trusty gatherings we tend to have the option to set up what some of the significant contemplations are tied in with receiving versatile Cloud Computing. With an unmistakable order knowledge, we will in general proceed with investigating and causing out polls to detect some of the courses through that the setup contemplations are regularly settled subsequently on blessing a feasible response to reestablishing trust in portable distributed computing, considering the tremendous edges of Mobile Cloud Computing and Cloud Computing; a premier examination group has tipped distributed computing that will be that the bedrock for Mobile Cloud Computing due to the first among the most elevated 10 most significant innovations with an improved possibility with time.

\section{Conclusion \& Future Research Scope}

It can be inferred from the detailed study of the models and the comparison table that the SmartLab architecture is the best of all the existing models. The Smartlab architecture offers good security, is low in cost, has a great future scope and offers max scalability among all 6 compared models. This paper researches the ideas of Mobile Cloud Computing (MCC), testing security issues and penetrates, different existing security systems, lastly, a few arrangements that expansion the security in the Mobile Cloud Environment. The majority of the systems neglected the security of client information protection, information stockpiling, and vitality safeguarding information sharing. Client information protection and mobile application that utilizations Cloud are the most testing factor. To accomplish greater security in the portable Cloud condition, dangers should be tended to and concentrated as needs be. To address all these security issues, the information security plan should be created which decreases the security dangers and furthermore reduces expenses and multifaceted nature to receive Cloud Computing in a portable domain. It is basic to remember that the structuring of things to come system arrangements ought to be more practical and ought to give better security and execution today.

\section{References}

[1] Schneider, "Essential characteristics of Mobile Cloud Computing", Marquette University, United States, 2012.

[2] Kun Yang, Dr. Shumao Ou, Hai Jin, Huazhong and Amiya Nayak, "Mobile Cloud Computing and Networking”, Proceedings of IEEE conference, 2013.

[3] M. Rajendra Prasad, Jayadev Gyani and P. R. K. Murti, "Mobile Cloud Computing: Implications and Challenges", Journal of Information Engineering and Applications", Vol 2, No.7, 2012, Print ISSN 2224-5782, pp. 7- 15.

[4] Ronnie D. Caytiles and Sunguk Lee, "Security Considerations for Public Mobile Cloud Computing", International Journal of Advanced Science and Technology, Vol. 44, July 2012.

[5] Soeung-Kon Victor Ko, Jung- Hoon Le and Sung Woo Kim, "Mobile Cloud Computing Security Considerations", April 30, 2012.

[6] Anand Surendra Shimpi and R. Chander, "Secure Framework in Data Processing for Mobile Cloud Computing", International Journal of Computer \& Communication Technology, ISSN (Print) 0975- 7449, vol. 3, Iss. 3, 2012.

[7] Jibitesh Mishra, Sanjit Kumar Dash and Sweta Dash, "Mobile Cloud Computing: A Secure Framework of Cloud Computing for Mobile Application", Institute for Computer Sciences, Social Informatics and Telecommunications Engineering, 2012, pp. $347-356$.

[8] Itani et al, "Towards Secure Mobile Cloud: A survey", Proceedings of Analyses paper, 2012.

[9] Eugene E. Marinelli, "Hyrax: Cloud Computing on Mobile Devices", Dissertation of Thesis, Carnegie Mellon University, Pittsburgh, 2009.

[10] Xiaojun Yu and Qiaoyan Wen, "Design of Security Solution to Mobile Cloud Storage",: Knowledge Discovery and Data Mining, AISC, Springer-Verlag Berlin Heidelberg H. Tan (Ed.), 2012, pp. 255-263.

[11] Robert Lemos, "Cloud's Future Security Depends on Mobile", Proceedings of RSA Conference, February 2012.

[12] V. L. Divya, "Mobile Applications with Cloud Computing”, International Journal of Scientific and Research, Vol. 2, Issue 4, April 2012, ISSN 2250-3153.

[13] Han Qi and Abdullah Gani, "Research on Mobile Cloud Computing: Trends, Review and Perspectives", Proceedings of Analyses paper, University of Malaya, Malaysia, 2012.

[14] S. Chetan, Gautam Kumar, K. Dinesh, Mathew K. and Abhimanyu M.A., "Cloud Computing for Mobile World", Proceedings of Analyses paper, National Institute of Technology, Calicut, 2010.

[15] Jon Oberheide and Evan Cooke, "Virtualized In-Cloud Security Services for Mobile Devices", Proceedings of the First Workshop on Virtualization in Mobile Computing, ACM, New York, USA, 2008, pp 31-35.

[16] "SmartLab Smartphone Programming Cloud Testbed". University of Cyprus. 
[17] Chang, V., Bacigalupo, D., Wills, G., and De Roure, D. A categorization of Cloud computing business models. In Proceedings of the 2010 10th IEEE/ACM International Conference on Cluster, Cloud and Grid Computing (pp. 509-512). IEEE Computer Society.

[18] Qian (Andy) Wang, "Mobile Cloud Computing", Master of Science Thesis, Department of Computer Science, University of Saskatchewan, Saskatoon, 2011.

[19] Soumya Ranjan Jena, and Zulfikhar Ahmad, "Response Time Minimization of Different Load Balancing Algorithms in Cloud Computing Environment”, IJCA, Vol 69, No. 17, Pages 22-27, May 2013.

[20] Soumya Ranjan Jena, and Bhushan Dewan, "Improving Quality-of-Service Constraints of Cloud Data Centers", IEEE, 2nd International Conference on Computing for Sustainable Global Development, BVICM, New Delhi ,2015.

[21] Soumya Ranjan Jena, Sudarshan Padhy, and Balendra Kumar Garg, "Performance Evaluation of Load Balancing Algorithms on Cloud Data Centers", IJSER, Vol 5, 3, Pages 1137-1145, 2014.

[22] Soumya Ranjan Jena, V. Vijayaraja, and Aditya Kumar Sahoo, "Performance Evaluation of Energy Efficient Power Models for Digital Cloud", INDJST, Vol 9, 48, Pages 1-7, 2016.

[23] Soumya Ranjan Jena, and L.Shridhara Rao,“ A Study on Energy Efficient Task Scheduler over Three-Tier Cloud Architecture using Green Cloud”, JARDCS, Vol 9, 18, pp. 727-735, 2017.

[24] Soumya Ranjan Jena, DR Lavanya, SS Gadde, "Minimization of execution time over Cloud computing environment using fuzzy technique", JARDCS, Vol 9, Issue 18, pp. 391-402, 2017,

[25] Soumya Ranjan Jena, Sukant Kishoro Bisoy and Bhushan Dewan, "Performance Evaluation of Energy Efficient Power Models for Differnent Scheduling Algorithms in Cloud using Cloud Reports", IEEE, GUCON 2019, International Conference on Computing, Power and Communication Technologies, pages. 880-891, Galgotias University, Greater Noida, U.P, India.

[26] Soumya Ranjan Jena, Raju Shanmugam, Rajesh Kumar Dhanaraj and Kavita Saini, "Recent Advances and Future Research Directions in Edge Cloud Framework", IJEAT, Volume 9, Issue 2, Pages. 439-444, December 2019.

[27] Soumya Ranjan Jena, Raju Shanmugam, Kavita Saini and Sanjay Kumar, "Cloud Computing Tools: Inside Views and Analysis", Proceedia of Computer Science, Volume 173, Pages. 382-391, 2020.

[28] Rajesh Kumar Dhanaraj, Soumya Ranjan Jena, Ashok Kumar Yadav, and Vani Rajasekar, "Mastering Disruptive TechnologiesApplications of Cloud Computing, IoT, Blockchain, Artificial Intelligence and Machine Learning Techniques", H.P. Hamilton, U.K, $1^{\text {st }}$ Edition, ISBN: 978-1913936235, 2021 (Book).

[29] S. N. Manoharan, Soumya Ranjan Jena, and A. Ilavendhan, "Euclidean Distance Based Similarity Measurement and Ensuing Ranking Scheme for Document Search from Outsourced Cloud Data", Turkish Journal of Computer and Mathematics Education, Volume 12, Issue No 3, pp. 4386-4395, 2021.

[30] Kiran, K.T.P.S., Satyanarayana, K.V.V., Yellamma, P., "Advanced Q-MAC: Optimal Resource Allocating for Dynamic Application in Mobile Cloud Computing using QoS with Cache Memory", International Journal of Engineering and Technology (UAE), Vol. 7, Special Issue, pp. 143-146, 2018.

[31] Pallavi, L., Jagan, A., Thirumala Rao, B., "ERMO Algorithm: An Energy Efficient Mobility Management in Mobile Cloud Computing System for 5G Heterogeneous Networks", International Journal of Electrical and Computer Engineering, Vol. 9, Issue 3, pp. 1957$1967,2019$.

[32] Pallavi, L., Jagan, A., Thirumala Rao, B., "BTS algorithm: An Energy Efficient Mobility Management in Mobile Cloud Computing System for 5G Heterogeneous Networks", Journal of Theoretical and Applied Information Technology, Vol. 97, Issue 1, pp. 48-60, 2019.

[33] Ramasubbareddy, S., Vedavasu, G., Gopi Krishna, K.B.N., Savithri, A., "PIOCM: Properly Identifying Optimized Cloudlet in Mobile Cloud Computing", Journal of Computational and Theoretical Nanoscience, Vol. 16, pp. 1967-1971, 2019.

[34] Sairam, V.P.N., Vurukonda, N., Likitha, K., Tarun, U., Deepak, V., "User Privacy-preserving Encryption Strategy out Sourcing Data in Mobile Cloud Computing", International Journal of Advanced Science and Technology, Vol. 29, Issue 7, pp. 739-746, 2020.

[35] Pallavi, L., Rao, B.T., Jagan, A., "Mobility Management Challenges And Solutions in Mobile Cloud Computing System for Next Generation Networks", International Journal of Advanced Computer Science and Applications, Vol. 11, Issue 3, pp. 177-192, 2020.

[36] Nalajala, S., Akhil, K., Sai, V., Shekhar, D.C., Tumuluru, P., "Light Weight Secure Data Sharing Scheme for Mobile Cloud Computing", Proceedings of the 3rd International Conference on I-SMAC IoT in Social, Mobile, Analytics and Cloud, I-SMAC 2019, pp. 613-617, 2019.

[37] Ravindranath, K., Rajasekhara Rao, K., "Dynamic Optimization Local Search Offloading Algorithm for Mobile Cloud Computing", International Journal of Applied Engineering Research, Vol. 9, Issue 14, pp. 2461-2472, 2014.

[38] Hoang T. Dinh, Chonho Lee, Dusit Niyato and Ping Wang, "A Survey of Mobile Cloud Computing: Architecture, Applications and Approaches, Wireless Communication and Mobile Computing”, 13, 1587-1611, 2013.

\section{Authors Profile}

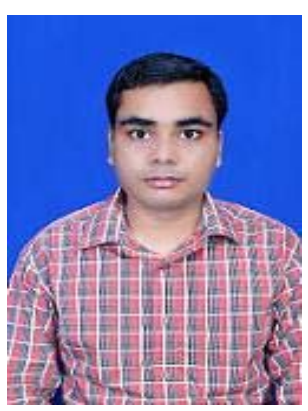

Mr. Soumya Ranjan Jena is currently working as an Assistant Professor in the Department of CSE, School of Computing at Vel Tech Rangarajan Dr. Sagunthala R\&D Institute of Science \& Technology, Avadi, Chennai, Tamil Nadu, India. He has teaching and research experience from various reputed institutions in India like Galgotias University, Greater Noida, Uttar Pradesh, AKS University, Satna, Madhya Pradesh, K L Deemed to be University, Guntur, Andhra Pradesh, GITA, Bhubaneswar, Odisha. He has been awarded M.Tech in IT, B.Tech in CSE, and Cisco Certified Network Associate (CCNA). He has got the immense experience to teach to graduate as well as postgraduate students and author of three books i.e. "Mastering Disruptive Technologies","Theory of Computation and Application" and "Design and Analysis of Algorithms". He has published more than 25 research papers on Cloud Computing, IoT in various international journals and conferences which are indexed by Scopus and Web of Science and also published three patents. 


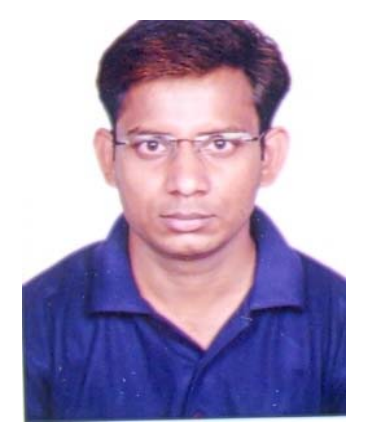

Mr. Ashok Kumar Yadav is currently working as Dean Academics and Assistant Professor at Rajkiya Engineering College, Azamgarh, Uttar Pradesh. He has worked as an Assistant Professor (on Ad-hoc) in the Department of Computer Science, University of Delhi. He has also worked with Cluster Innovation Center, University of Delhi, New Delhi. He qualified for UGC-JRF. Presently, he is currently pursuing his Ph.D. in Computer Science from JNU, New Delhi. He has received M.Tech in Computer Science and Technology from JNU, New Delhi. He has presented and published papers at international conferences and journals on blockchain technology and machine learning. He has delivered various expert lectures on reputed institutes.

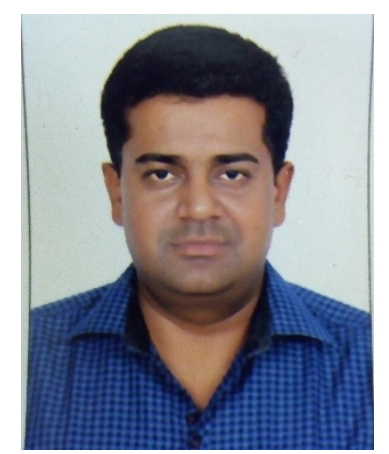

Mr. Shrikant Patel is working as an Assistant Professor in Computer Science and Application department at Delhi Skill and Entrepreneurship University, Delhi (Govt. of NCT of Delhi), India. He has more than 18 years of teaching experience in various leading institutes/organizations at both UG \& PG levels. He is an alumnus of Dr. Hari Singh Gour Central University, Sagar MP. He has completed his MCA and M. Phil in Computer Science from DHSGU Sagar, MP. He is an UG topper and Gold Medalist in Dr. Hari Singh Gour University, Sagar, MP. He has published about more than 15 research papers in various national and international journals of repute. Moreover he has guided more than 200 students for their minor and major research projects in various subfield of Computer Science and Information Technology. His current research interest are Software Engineering, Machine Learning and IoT. He is also a life member of Indian Society for Technical Education.

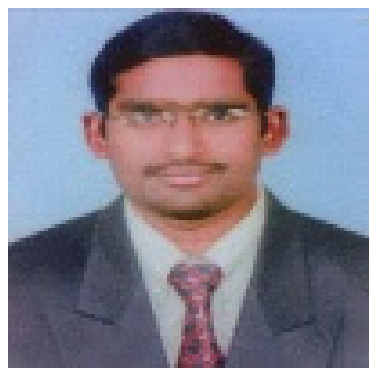

Mr. CH. M. H. Saibaba working presently as Assistant Professor in the Department of Computer Science \& Engineering at Koneru Lakshmaiah Education Foundation, Vaddeswaram, Guntur, Andhra Pradesh. He has more than 19 years of teaching experience in various reputed institutions in Andhra Pradesh. Currently, he is pursuing Ph.D. in SCSVMV University, Kanchipuram. He has completed M.Tech from Bharathidasan University and M.Sc (Computer Science) from Kakatiya University. He has published 19 Journal papers in various reputed National and International journals. His research interests include Data Science, Data Warehousing and Data Mining. 